\title{
Effect of alloy non-uniformity on optical properties of InGaN bulk and quantum wells
}

\author{
A. Di Vito ${ }^{\dagger}$, A. Pecchia ${ }^{\ddagger}$, A. Di Carlo ${ }^{\dagger}$ \\ M. Auf der Maur ${ }^{\dagger, *}$, \\ †Dept. of Electronic Engineering, Università degli Studi di Roma "Tor Vergata”, Italy \\ ${ }^{\ddagger}$ CNR-ISMN, via Salaria Km 29.300, 00017 Monterotondo, Rome (Italy) \\ *Email: auf.der.maur@ing.uniroma2.it
}

\begin{abstract}
In this work we present a theoretical study of the effects of nanometer-scale non-uniformities in InGaN alloys on their optical properties. We show by empirical tight-binding calculations that, compared to a random alloy, deviations from uniformity lead to an increasing scattering of both the optical matrix elements and the emission energy. While the extracted Bparameter of radiative recombination appears to be relatively insensitive to small non-uniformities, at larger degree of In clustering we can demonstrate the transition to quantum dot like behaviour. Our results show that alloy non-uniformity allows to reproduce both the measured spectral width of electroluminescence spectra and temperature behaviour of the radiative recombination parameter.
\end{abstract}

\section{INTRODUCTION}

Light Emitting Diodes (LEDs) based on Indium Gallium Nitride (InGaN) were first demonstrated by Nakamura et al. in 1993. Currently, they are the most commercially successful devices for high efficiency solid state lighting (SSL) applications [1], [2], [3]. As a matter of fact, InGaN-based LEDs can potentially cover the whole visible spectrum, thus allowing in principle to eliminate the phosphors used in white LEDs for down conversion [4], [5]. Both experimental measurements and theoretical studies have drawn the attention on the presence and the effect of statistical fluctuations of the InGaN alloy composition on the nanometer scale [6], [7], [8], [9]. Despite the high number of studies conducted so far, the impact of In clustering on the optical properties of realistic InGaN/GaN Quantum Well (QW) LEDs has not yet been discussed in detail. Furthermore, the theoretical analyses based on ab-initio approaches performed on InGaN bulk structures lack a properly large supercell size and a significantly high number of random samples simulations.

In the present work, we theoretically calculated the spontaneous emission spectrum, the ground state (GS) optical transitions momentum matrix elements (MME) and the radiative recombination coefficient $B$ for bulk $\mathrm{InGaN}$ and $\mathrm{InGaN} / \mathrm{GaN}$ single QW (SQW) LEDs taking into account random fluctuations of In content within the well with several degrees of In clustering. For the simulations we use an empirical tight-binding model (ETB) with $\mathrm{sp}^{3} \mathrm{~d}^{5} \mathrm{~s}^{*}$ parametrization [10]. The relaxed atomistic structure has been calculated by a modified Keating's valence force field (VFF) model [11]. The quality of the modeling approach has been tested by direct comparison with density functional theory (DFT) in local density approximation (LDA) on smaller bulk structures.

We assumed a single QW LED with AlGaN electron blocking layer as in [8], and with $20 \%$ of Indium in the QW. The atomistic structure was created including the $3 \mathrm{~nm}$ thick QW and $3 \mathrm{~nm}$ of $\mathrm{GaN}$ barrier on both sides. In order to be able to describe localization of the carriers, we used a supercell size in the QW plane of $10 \times 10 \mathrm{~nm}$. The calculations have been performed at a realistic operating point near peak internal quantum efficiency, in order to be able to compare results with measurement data.

For the construction of the random non-uniform InGaN alloy we used the following approach. We first create a pure GaN structure and calculate the number $N_{\text {In }}$ of Indium atoms to be substituted in order to obtain the required mean $\mathrm{In}_{x} \mathrm{Ga}_{1-x} \mathrm{~N}$ composition. Then we randomly substitute a fraction $y$ of $N_{\text {In }}$ with uniform probability $x$. Note that if $y=1$ a uniform random alloy results. Subsequently, we substitute the remaining Indium atoms, but this time the probability of changing a Ga atom into an Indium atom is proportional to the number of Indium atoms already present up to the second nearest cation site, producing thus a spatially non-uniform probability. For our study we used $y=0.8,0.6$ and 0.4 .

For each degree of clustering, indexed by $y$, we performed simulations on 50 random samples, allowing to calculate statistically meaningful mean values and the global emission spectra to be compared with experimental data.

\section{Simulation RESUltS}

We calculated the first 8 electron and hole states and the different optical matrix elements for both bulk InGaN and a $3 \mathrm{~nm}$ QW with $20 \%$ of Indium content, and with increasing degree of clustering corresponding to seed values $y$ of $0.8,0.6$ and 0.4. The electrostatic potential in the QW case has been obtained from a self-consistent Schrödinger/driftdiffusion calculation at the peak efficiency. Figure 1a shows the cumulative density of states (DOS) obtained by summing the DOS of all random samples for each degree of clustering. We observe the formation of band tails both in the valence and in the conduction band. These tails result in a broadening of the emission spectra towards low energies, as shown in Fig. 1 b. Note that the measured spectrum in Fig. 1b (black line) is from a SQW LED with nominally $19.5 \%$ Indium, but identical 


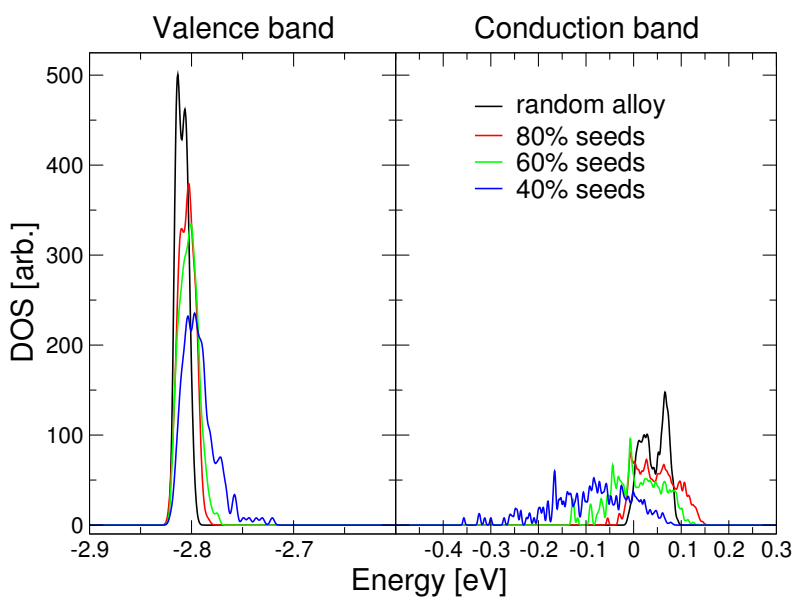

(a)

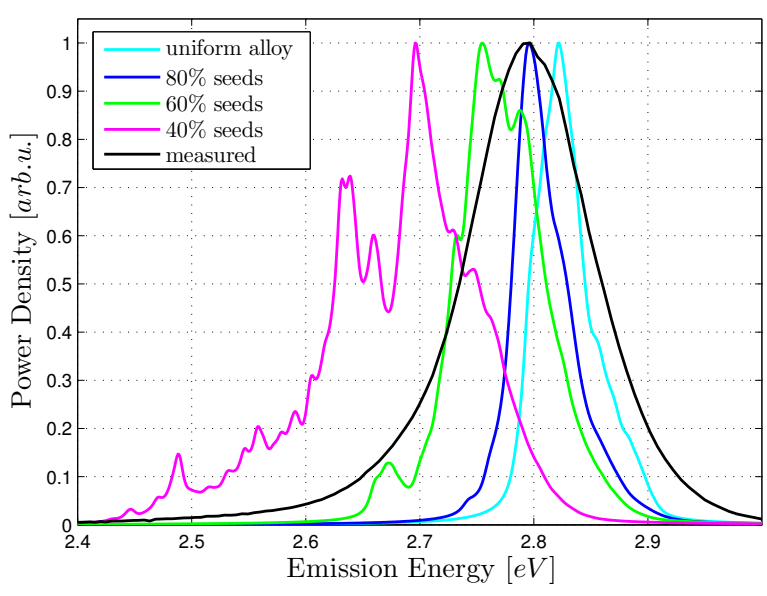

(b)

Fig. 1. Cumulative density of states for random alloy and different degree of clustering (a), and corresponding predicted electroluminescence spectra (b).

structure otherwise. It can be seen that a certain degree of non-uniformity can reasonably reproduce the observed spectral width. The non-uniform alloy resulting from $y=0.4$ starts to show quantum dot (QD) like features, with prominent peaks in the spectrum. Figure 2 shows the histograms of the radiative recombination parameter extracted from the QW calculations. The total recombination $R$ is obtained as the mean of all random samples, and $B$ is then calculated from $R=B \bar{n} \bar{p}$, where $\bar{n}$ and $\bar{p}$ are the mean carrier densities. From the figure we can see the increasing scattering in $B . B$ remains unchanged for small degree of clustering, while for larger degree $(y=0.4)$ it starts to increase, since indium-rich regions start to act as QDs, localizing both holes and electrons in the same spatial position.

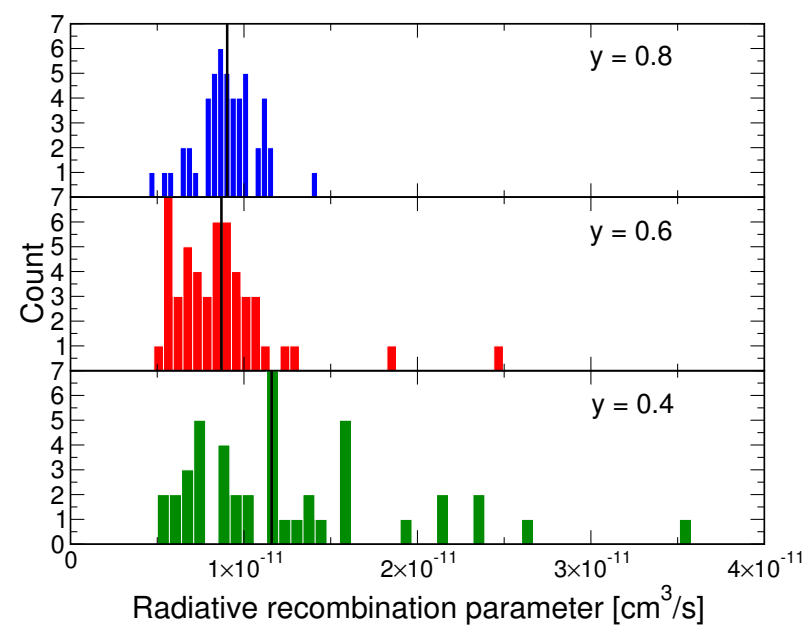

Fig. 2. Histograms for the radiative recombinatin parameter $B$ for different degrees of non-uniformity. The mean value is shown with a vertical black bar.

\section{ACKNOWLEDGMENT}

This work has been supported by Horizon 2020 project ChipScope, under grant agreement number 737089.

\section{REFERENCES}

[1] S. P. DenBaars, D. Feezell, K. Kelchner, S. Pimputkar, C.-C. Pan, C.-C. Yen, S. Tanaka, Y. Zhao, N. Pfaff, R. Farrell, M. Iza, S. Keller, U. Mishra, J. S. Speck, and S. Nakamura, "Development of gallium-nitride-based light-emitting diodes (leds) and laser diodes for energy-efficient lighting and displays," Acta Materialia, vol. 61, no. 3, pp. 945 - 951, 2013, the Diamond Jubilee Issue. [Online]. Available: http://www.sciencedirect.com/science/article/pii/S1359645412007896

[2] S. Nakamura and M. R. Krames, "History of gallium nitride-based lightemitting diodes for illumination," Proceedings of the IEEE, vol. 101, no. 10, pp. 2211-2220, Oct 2013.

[3] K. Hwa-Mok, C. Yong-Hoon, L. Hosang, K. Suk Il, R. Sung Ryong, K. Deuk Young, K. Tae Won, and C. Kwan Soo, "High-Brightness Light Emitting Diodes Using Dislocation-Free Indium Gallium Nitride/Gallium Nitride Multiquantum-Well Nanorod Arrays," Nano Lett., vol. 4, p. 1059, 2004.

[4] Hon-Way Lin, Yu-Jung Lu, Hung-Ying Chen, Hong-Mao Lee, and Shangjr Gwo, "InGaN/GaN nanorod array white light-emitting diode," Appl. Phys. Lett., vol. 97, pp. 073-101, 2010.

[5] Light-emitting diodes for solid-state lighting: searching room for improvements, vol. 9768, 2016. [Online]. Available: https://doi.org/10.1117/12.2207265

[6] R. Butté, L. Lahourcade, T. K. Uzdavinys, G. Callsen, M. Mensi, M. Glauser, G. Rossbach, D. Martin, J.-F. Carlin, S. Marcinkevicius, and N. Grandjean, "Optical absorption edge broadening in thick ingan layers: Random alloy atomic disorder and growth mode induced fluctuations," Applied Physics Letters, vol. 112, no. 3, p. 032106, 2018.

[7] C. M. Jones, C.-H. Teng, Q. Yan, P.-C. Ku, and E. Kioupakis, "Impact of carrier localization on recombination in ingan quantum wells and the efficiency of nitride light-emitting diodes: Insights from theory and numerical simulations," Applied Physics Letters, vol. 111, no. 11, p. 113501, 2017.

[8] M. Auf der Maur, A. Pecchia, G. Penazzi, W. Rodrigues, and A. Di Carlo, "Efficiency Drop in Green InGaN/GaN Light Emitting Diodes: The Role of Random Alloy Fluctuations," Physical Review Letters, vol. 116, no. 2, JAN 152016.

[9] Carrier localization in InGaN-based light-emitting diodes (Conference Presentation), vol. 10554, 2018.

[10] J. M. Jancu, F. Bassani, F. Della Sala, and R. Cholz, "Transferable tight-binding parametrization for the group-iii nitrides," Applied Physics Letters, vol. 81, no. 25, p. 4838, 2002.

[11] D. Camacho and Y. M. Niquet, "Application of keating's valence force field model to non-ideal wurtzite materials," Physica E, vol. 42, pp. 1361-1364, 2010. 